\title{
A NOTE ON A THEOREM OF GANEA, HILTON AND PETERSON
}

\author{
C. S. HOO
}

Introduction. Let $X$ be a space. We are interested in the question whether or not the loop space $\Omega X$ and the suspension $\Sigma X$ are homotopy commutative, that is whether or not nil $X \leqq 1$, conil $X \leqq 1$ respectively. Let $i: X b X \rightarrow X \vee X$ be the fibre of the inclusion $j: X \vee X$ $\rightarrow X \times X$. Let $\nabla: X \vee X \rightarrow X$ be the folding map. Then in [3], Ganea, Hilton and Peterson proved the following

THEOREM 1. Let $X$ be 1-connected. Then nil $X \leqq 1$ if and only if $\nabla i=0$.

Dually, let $q: X \times X \rightarrow X \wedge X$ be the cofibre of the inclusion $j$, and let $\triangle: X \rightarrow X \times X$ be the diagonal map. Let $e^{\prime}: X \wedge X \rightarrow \Omega \Sigma(X \wedge X)$ be the canonical imbedding. Then in [3], the authors also proved

Theorem 2. Let $X$ be o-connected. Then conil $X \leqq 1$ if and only if $e^{\prime} q \triangle=0$.

This paper represents an attempt to understand these theorems. Let $c: \Omega(X \bigvee X) \times \Omega(X \vee X) \rightarrow \Omega(X \bigvee X)$ be the commutator map. We shall define below a map $\bar{c}: \Sigma(\Omega X \times \Omega X) \rightarrow X \vee X$ obtained from $c$. Applying the co-Hopf construction, we have a map $H(\bar{c}): \Omega \Sigma(\Omega X \times \Omega X)$ $\rightarrow \Omega(X b X)$. Then we prove

Theorem 3. $c=\Omega(\nabla i) H(\bar{c}) e^{\prime}: \Omega X \times \Omega X \rightarrow \Omega X$, the commutator map.

We observe, of course, that the condition for nil $X \leqq 1$ is precisely $c=0$. Dually, let $c^{\prime}: \Sigma(X \times X) \rightarrow \Sigma(X \times X) \vee \Sigma(X \times X)$ be the cocommutator map. This gives a map $\bar{c}^{\prime}: X \times X \rightarrow \Omega(\Sigma X \vee \Sigma X)$. The Hopf construction then gives a map $J\left(\bar{c}^{\prime}\right): \Sigma(X \wedge X) \rightarrow \Sigma \Omega(\Sigma X \bigvee \Sigma X)$. Let $e: \Sigma \Omega(\Sigma X \bigvee \Sigma X) \rightarrow \Sigma X \bigvee \Sigma X$ be the map having $1_{\Omega(\Sigma X \vee \Sigma X)}$ as its adjoint. Let us denote the cocommutator product $\Sigma X \rightarrow \Sigma X \vee \Sigma X$ by $c^{\prime}$ also. The condition for conil $X \leqq 1$ is precisely $c^{\prime}=0$. We prove

TheOREM 4. $c^{\prime}=e J\left(\bar{c}^{\prime}\right) \Sigma(q \triangle): \Sigma X \rightarrow \Sigma X \bigvee \Sigma X$.

We work in the category of spaces with base point and having the homotopy type of countable $\mathrm{CW}$-complexes. For simplicity, we shall frequently use the same symbol for a map and its homotopy class.

Received by the editors April 18, 1967. 
1. Let $A, B$ be spaces. We have the fibration $A$ b $B \stackrel{i}{\rightarrow} A \vee B$ $\stackrel{\lrcorner}{\rightarrow} A \times B$. We can find a map $\chi: \Omega(A \times B) \rightarrow \Omega(A \vee B)$ such that $(\Omega j) \chi \simeq 1_{\Omega(A \times B)}$. In fact we can take $\chi=\Omega\left(i_{A} p_{A}\right)+\Omega\left(i_{B} p_{B}\right)$ where $p_{A}, p_{B}$ are the projections of $A \times B$ onto the factors and $i_{A}: A \rightarrow A \vee B$, $i_{B}: B \rightarrow A \bigvee B$ are the inclusions. The exact sequence of the fibration now shows that there exists a unique element $[g] \in[\Omega(A \vee B)$, $\Omega(A$ b $B)]$ such that $1_{\Omega(A \bigvee B)}=(\Omega i) g+\chi(\Omega j)$.

Now for any space $X$ and a map $f: X \rightarrow A \vee B$ we can form the map $H(f)=g(\Omega f): \Omega X \rightarrow \Omega(A b B)$. We shall call this the co-Hopf construction. The element $[H(f)]$ is the unique element of $[\Omega X, \Omega(A b B)]$ satisfying $[\Omega f]=(\Omega i)_{\sharp}[H(f)]+[\chi \Omega(j f)]=(\Omega i)_{\sharp}[H(f)]+\left[\Omega\left(i_{A} \pi_{A} f\right)\right]+$ $\left[\Omega\left(i_{B} \pi_{B} f\right)\right]$ where $\pi_{A}: A \vee B \rightarrow A, \pi_{B}: A \vee B \rightarrow B$ are induced by the projections onto the factors.

For spaces $X, Y$ we have a bijection $\tau:[\Sigma X, Y] \rightarrow[X, \Omega Y]$ which takes each map to its adjoint. Suppose $X$ is a given space. We have a projection $p: \Sigma \Omega X \rightarrow X$ such that $\tau(p)=1_{\Omega X}$. Let $p_{1}=i_{1} p, p_{2}=i_{2} p$ where $i_{1}, i_{2}: X \rightarrow X \vee X$ are the injections in the first and second copies of $X$ respectively. Let $c: \Omega(X \bigvee X) \times \Omega(X \vee X) \rightarrow \Omega(X \vee X)$ be the commutator map. Then we can form the map $\bar{c}=\tau^{-1}\left\{c\left(\tau\left(p_{1}\right) \times \tau\left(p_{2}\right)\right)\right\}$ : $\Sigma(\Omega X \times \Omega X) \rightarrow X \bigvee X$. It is now easily verified that $\nabla \bar{c}=\tau^{-1}(c)$. The co-Hopf construction, applied to $\bar{c}$, gives an element $H(\bar{c}): \Omega \Sigma(\Omega X \times \Omega X)$ $\rightarrow \Omega(X b X)$. Let $e^{\prime}: \Omega X \times \Omega X \rightarrow \Omega \Sigma(\Omega X \times \Omega X)$ be such that $e^{\prime}=\tau\left(1_{\Sigma(\Omega X \times \Omega X)}\right)$. It is easily seen that $\Omega\left(\tau^{-1}(c)\right) e^{\prime}=c: \Omega X \times \Omega X \rightarrow \Omega X$, the commutator map. Since $\nabla \bar{c}=\tau^{-1}(c)$, Theorem 3 follows immediately from

THEOREM 5. $\Omega(\nabla \bar{c})=\Omega(\nabla i) H(\bar{c}): \Omega \Sigma(\Omega X \times \Omega X) \rightarrow \Omega X$.

Proof. $H(\bar{c})$ satisfies $\Omega \bar{c}=(\Omega i) H(\bar{c})+\Omega\left(i_{1} \pi_{1} \bar{c}\right)+\Omega\left(i_{2} \pi_{2} \bar{c}\right)$ where $\pi_{1}, \pi_{2}: X \bigvee X \rightarrow X$ are induced by the projections onto the factors, and $i_{1}, i_{2}: X \rightarrow X \vee X$ are the imbeddings in the first and second copies of $X$ respectively. We have $\Omega(\nabla \bar{c})=\Omega(\nabla i) H(\bar{c})+\Omega\left(\nabla i_{1} \pi_{1} \bar{c}\right)+\Omega\left(\nabla i_{2} \pi_{2} \bar{c}\right)$. Let $\phi$ be the loop multiplication on $\Omega X$ and $\mu$ the loop inverse. Then a simple check shows that $\tau\left(\nabla i_{1} \pi_{1} \bar{c}\right)=\phi\left\{\phi\left(1 X^{*}\right) \triangle X \phi\left(1 \times^{*}\right) \triangle \mu\right\} \triangle r_{1}$ where $\triangle$ is the diagonal map and $r_{1}: \Omega X \times \Omega X \rightarrow \Omega X$ is the projection onto the first factor. Since $\phi\left(1 X^{*}\right) \triangle \simeq 1$ and $\phi(1 \times \mu) \triangle \simeq^{*}$, we have $\tau\left(\nabla i_{1} \pi_{1} \bar{c}\right)=0$. Hence $\nabla i_{1} \pi_{1} \bar{c}=0$. Similarly $\nabla i_{2} \pi_{2} \bar{c}=0$. It follows then that $\Omega(\nabla \bar{c})=\Omega(\nabla i) H(\bar{c})$.

Theorems 1 and 3 are the immediate

Corollary. Let $X$ be 1-connected. If $\Omega(\nabla i)=0$, then $\nabla i=0$.

REMARK. In [3], it is shown that there exist maps $a, b$ such that $b a=1, i b=\bar{c}$. It is clear from the above that $H(\bar{c})=\Omega b$. 
2. We now dualise. Let $p_{1}, p_{2}: X \times X \rightarrow X$ be the projections, and let $e_{i}=e^{\prime} p_{i}$ where $e^{\prime}: X \rightarrow \Omega \Sigma X$ is the canonical imbedding. Let $c^{\prime}$ be the cocommutator map $\Sigma(X \times X) \rightarrow \Sigma(X \times X) \vee \Sigma(X \times X)$. Let $\bar{c}^{\prime}$ $=\tau\left\{\left(\tau^{-1}\left(e_{1}\right) \vee \tau^{-1}\left(e_{2}\right)\right) c^{\prime}\right\}: X \times X \rightarrow \Omega(\Sigma X \vee \Sigma X)$. Then $\bar{c}^{\prime} \Delta=\tau\left(c^{\prime}\right)$ where $\triangle$ is the diagonal map.

Let $A, B$ be spaces. We consider the cofibration $A \vee B \stackrel{\jmath}{\rightarrow} A \times B$ $\stackrel{q}{\rightarrow} A \wedge B$. There exists a map $p: \Sigma(A \times B) \rightarrow \Sigma(A \vee B)$ such that $p(\Sigma j) \simeq 1_{\Sigma(A \vee B)}$. The exact sequence of the cofibration now shows that $(\Sigma q)^{\#}$ is a monomorphism. Dual to the above, we now see that there exists a unique element $[d] \in[\Sigma(A \wedge B), \Sigma(A \times B)]$ satisfying $1_{\Sigma(A \times B)}=d(\Sigma q)+(\Sigma j) p$.

Given a map $f: A \times B \rightarrow X$ we can now define $J(f)=(\Sigma f) d: \Sigma(A \wedge B)$ $\rightarrow \Sigma X$. We shall call $J(f)$ the map obtained from $f$ by the Hopf construction. The element $[J(f)]$ is the unique element satisfying $[\Sigma f]$ $=(\Sigma q)^{\sharp}[J(f)]+[\Sigma(f j) p]=(\Sigma q)^{\sharp}[J(f)]+\left[\Sigma\left(f j p_{A}\right)\right]+\left[\Sigma\left(f j p_{B}\right)\right]$ where $p_{A}, p_{B}: A \times B \rightarrow A \vee B$ are induced by the projections onto the first and second coordinates respectively. We can now consider the element $J\left(\bar{c}^{\prime}\right): \Sigma(X \wedge X) \rightarrow \Sigma \Omega(\Sigma X \bigvee \Sigma X)$. We have $J\left(\bar{c}^{\prime}\right) \Sigma(q \triangle), \Sigma\left(\bar{c}^{\prime} \triangle\right)$ : $\Sigma X \rightarrow \Sigma \Omega(\Sigma X \vee \Sigma X)$. Let $e: \Sigma \Omega(\Sigma X \vee \Sigma X) \rightarrow \Sigma X \vee \Sigma X$ be such that $\tau(e)=1_{\Omega(\Sigma X \vee \Sigma X)}$. Let $c^{\prime}: \Sigma X \rightarrow \Sigma X \vee \Sigma X$ be the cocommutator map. It is now easily checked that $e \Sigma\left(\tau\left(c^{\prime}\right)\right)=c^{\prime}$. Since $\bar{c}^{\prime} \Delta=\tau\left(c^{\prime}\right)$. Theorem 4 follows immediately from

Theorem 6. $\Sigma\left(\bar{c}^{\prime} \triangle\right)=J\left(\bar{c}^{\prime}\right) \Sigma(q \triangle): \Sigma X \rightarrow \Sigma \Omega(\Sigma X \bigvee \Sigma X)$.

Proof. The proof is completely dual to that of Theorem 5 , and we shall omit it.

REMARK 1. In [3], it is shown that we can find maps $a^{\prime}, b^{\prime}$ such that $b^{\prime} a^{\prime}=1, a^{\prime} e^{\prime} q=\bar{c}^{\prime}$. It is easily seen that $J\left(\bar{c}^{\prime}\right)=\Sigma\left(a^{\prime} e^{\prime}\right)$.

REMARK 2. Theorems 3 and 4 give other conditions for nil $X \leqq 1$, conil $X \leqq 1$ respectively, namely whenever some combination of factors in the factorizations of $c, c^{\prime}$ is null-homotopic.

\section{REFERENCES}

1. I. Berstein and T. Ganea, Homotopical nilpotency, Illinois J. Math. 5 (1961), 99-129.

2. I. Berstein and P. J. Hilton, On suspensions and comultiplications, Topology 2 (1963), 73-82.

3. T. Ganea, P. J. Hilton and F. P. Peterson, On the homotopy-commutativity of loop-spaces and suspension, Topology 1(1962), 133-141.

4. P. J. Hilton, Homotopy theory and duality, Gordon and Breach, New York, 1965.

University of Alberta 